\title{
EXTENSIÓN CULTURAL EN LA UNIVERSIDAD DE TALCA: ANTECEDENTES, REALIDAD PRESENTE Y DESAFÍOS
}

\author{
Pedro E. Zamorano*
}




\section{RESUMEN}

Después de un rápido recorrido por la historia de la vida y la actividad cultural universitarias en la ciudad chilena de Talca, este artículo recoge las principales iniciativas de extensión cultural que realiza, en los más variados ámbitos del arte, la Universidad de Talca, todas ellas basadas en los principios de excelencia, tolerancia, amplitud ideológico-estética y responsabilidad social. Destaca que gran parte de lo obrado en materia cultural apunta a revertir los fenómenos del centralismo cultural -que estructura una desigual y creciente inequidad en el acceso a la cultura-, y al esfuerzo corporativo por compensar el déficit de capital cultural con que ingresa un alto porcentaje de los estudiantes universitarios.

\section{ABSTRACT}

After a quick review of the history, life and cultural activities at the university in the Chilean city of Talca, this paper describes the main cultural outreach activities carried out by the Universidad de Talca in different artistic fields, all of which are based on the principles of excellence, tolerance, broadmindedness in ideological and esthetical terms and social responsibility. The paper emphasizes the fact that a large part of what has been accomplished in cultural terms is intended to revert the phenomenon of cultural centralism -that gives rise to an unequal and growing inequality in the access to culture-and the corporate efforts to compensate for the lack of cultural capital with which a high percentage of university students enroll in the university. 


\section{EXTENSIÓN CULTURAL EN LA UNIVERSIDAD DE TALCA: ANTECEDENTES, REALIDAD PRESENTE Y DESAFÍOS}

\section{EL CONTEXTO ORIGINARIO}

En los inicios de la década de los sesenta se fundaron en Talca los primeros proyectos universitarios. En octubre de 1962, la Universidad Técnica del Estado creó un Centro Universitario para satisfacer las necesidades de enseñanza superior en la zona ${ }^{1}$. En forma paralela se efectuaban gestiones para la creación de un Colegio Regional Universitario dependiente de la Universidad de Chile, dirigida en ese entonces por el rector Juan Gómez Millas. Con tal motivo se constituyó un comité denominado "Pro-Colegios Regionales Universitarios en Talca”, el que tuvo como misión reunir, por medio de erogación pública de la comunidad, la suma de cien millones de escudos ${ }^{2}$. El esfuerzo de los talquinos tenía plena justificación; en aquella época las oportunidades para la juventud de la zona eran escasas. Seguir estudios profesionales en Santiago o Concepción era la opción de quienes tenían talento y recursos, pero muchos no regresaban, produciéndose una importante pérdida de inteligencia, clave para cualquier proceso de desarrollo.

1 El 17 de octubre de 1962 se creó el Centro Universitario de Talca, dependiente de la Universidad Técnica del Estado, inaugurado oficialmente el 14 de diciembre, por el rector de la Universidad, don Horacio Aravena Andaur. En la ocasión se nombró como director en Talca a don Samuel Jiménez Sepúlveda.

2 Este monto era el aporte que condicionaba la rectoría de la Universidad de Chile a la petición que se formulara sobre la fundación del Colegio Universitario de Talca. 
Los proyectos universitarios, que luego dan origen a las sedes de la Universidad Técnica del Estado y de la Universidad de Chile, abrieron nuevos espacios y posibilidades para la juventud de la zona. La presencia de las dos principales universidades del país se concretó en carreras de nivel técnico, algunas pedagogías, más algunas carreras del área de la salud. También comenzaron a desarrollarse incipientes programas de extensión cultural, que se relacionaron básicamente con exposiciones de pintura, algunas conferencias a cargo de académicos de Santiago y, ocasionalmente, alguna actividad musical. Estas sedes universitarias, entonces, aun cuando desarrollaron proyectos fundamentalmente docentes, fueron creando de forma paulatina espacios para la reflexión y la cultura.

Talca exhibía por aquella época una actividad cultural decadente. Del otrora tradicional Teatro Municipal de la ciudad, por donde habían desfilado importantes artistas y elencos, quedaban sólo recuerdos y un proyecto de reconstrucción, hasta hoy inconcluso, financiado por la ley conocida como de "el fósforo".

El debate local tenía su espacio en el diario La Mañana, medio que, ya desde la mitad del siglo XX, había posicionado en el imaginario colectivo de los talquinos temas tan importantes como el puerto de Constitución, el Paso Pehuenche y la creación de la universidad. El compromiso con la cultura y con estos ideales colectivos había sido estimulado por el director y propietario de este medio, Juan C. Bravo, y por la pluma del cronista Benito Riquelme González, quien, bajo el pseudónimo de "Rigón Benoit", siguió el acontecer cultural de la ciudad por más de treinta años a través de sus "Crónicas talquinas".

La actividad pictórica era promovida por el Centro de Amigos del Arte, que realizaba clases, exposiciones y salones locales, algunas veces con la concurrencia de artistas nacionales vinculados, preferentemente, a la Universidad de Chile.

En términos generales, podemos hablar de una cultura más bien autista, generada por acciones espontáneas de un voluntariado integrado por personas sensibles y cultas, y sin apoyos institucionales, caracterizada, además, por la ausencia de referentes críticos y por la 
escasez de circuitos para su difusión. No había instancias para la formación de profesionales de la cultura y el patrimonio era más que escaso.

El capítulo más alto en el desarrollo de la cultura local fue quizás el que escribió, en el Liceo de Hombres de la ciudad, un conjunto de intelectuales y hombres públicos de figuración nacional, a los que el diplomático y escritor talquino Oscar Pinochet de la Barra denomina como "Generación del Centenario".

El legado dejado en la entidad por el rector Enrique Molina Garmendia y por su vicerrector Alejandro Venegas Carus ${ }^{3}$-más conocido como el doctor Julio Valdés Cange-, había alentado inquietudes intelectuales en numerosos jóvenes, entre ellos Ricardo Donoso, Max Jara, Domingo Melfi, además de quienes formaron luego el grupo "La Mandrágora" -quizá el exclusivo testimonio surrealista en Chile por aquella época-, integrado por los literatos Enrique Gómez Correa, Braulio Arenas, Teófilo Cid, además del pintor Eugenio Vidaurrázaga Concha.

La presencia universitaria produjo un positivo impacto en la ciudad y en la región. Aparte de dar posibilidades de formación profesional a la juventud de la zona, promovió un movimiento importante de profesionales, muchos de ellos académicos, entre Santiago y la ciudad. Generó también una masa estudiantil procedente, en buena parte, de otros lugares del país.

Las sedes llegaron a tener una matrícula superior a diez mil alumnos, que dieron otra fisonomía cultural a Talca, a la vez que otras oportunidades económicas a muchas familias. Se desarrollan pequeñas empresas relacionadas con los distintos servicios universitarios.

La extensión cultural formó parte importante de las acciones desarrolladas hacia la comunidad por estas sedes. La Universidad

3 Enrique Molina Garmendia y Alejandro Venegas llegaron a principios de siglo al Liceo de Hombres de Talca, el primero como rector y el segundo como vicerrector. Molina Garmendia llegó a ser fundador y primer Rector de la Universidad de Concepción, en tanto que Venegas, más conocido por el pseudónimo de "Dr. Julio Valdés Cange", conoció el castigo de la sociedad chilena y luego el olvido, quizá por su polémica obra "Sinceridad". 
Técnica del Estado tuvo un programa de exposiciones pictóricas y creó una radio con programación preferentemente cultural. La sede de la Universidad de Chile tenía un Departamento de Arte, del cual dependían las carreras de Pedagogía en Artes Plásticas y Pedagogía en Educación Musical. Desde éstas se generaron programas de exposiciones, ciclos de conciertos y un coro universitario.

La creación, en 1981, del Instituto Profesional de Talca, que dio origen, luego, a la Universidad de Talca, fusiona a estas sedes universitarias en un solo proyecto académico. Esta autonomía definió nuevos desafíos y nuevas relaciones con el entorno profesional, social y cultural. La universidad, de este modo, redefinió su proyecto académico en un marco de nuevas demandas, propiciando relaciones más dinámicas y estrechas con el medio externo.

En el contexto de la extensión cultural, en una primera etapa, consolidó su programa de exposiciones artísticas, sus ciclos de conciertos y presentaciones de teatro, creó un conjunto folclórico y un taller de teatro, a la vez que mantuvo su coro y radio universitarios.

Con más de cuarenta años de tradición universitaria en la ciudad y la región y con un proyecto universitario autónomo de más de veinte años, la Universidad de Talca ha experimentado progresos significativos en todas sus facetas, que la sitúan en un plano de distinción en el escenario de la educación superior nacional. La estrategia de desarrollo institucional ha privilegiado la calificación de los recursos humanos, la inversión en tecnología e infraestructura, la vinculación internacional y la inversión en estudiantes, todo lo cual ha hecho posible lograr mejores estándares de excelencia en la docencia, la investigación, la transferencia tecnológica y en las actividades de extensión académica y cultural.

\section{EXTENSIÓN CULTURAL: ALGUNOS CRITERIOS Y PRINCIPIOS}

Situada en un escenario universitario, en este caso de una institución pública y regional, la extensión cultural está representada por principios y exigencias de calidad, tolerancia, amplitud ideológicoestética y responsabilidad social. 
La excelencia se manifiesta como un imperativo en todas las actividades desarrolladas por la institución. Se expresa como un valor inmanente, ético y como una aspiración en la gestión.

La tolerancia y la flexibilidad ideológico-estética dicen relación con el planteamiento democrático inherente al fenómeno artístico contemporáneo. Como institución de educación, la universidad debe acoger en sus propuestas culturales la más amplia diversidad ideológica y estética que se da en las expresiones del arte y la cultura: lo clásico, la vanguardia y los distintos lenguajes y momentos del arte.

Por otra parte, la responsabilidad social está vinculada con el carácter público de la institución. Dada su naturaleza, debe promover la igualdad de oportunidades y el acceso equitativo a los bienes de la cultura. El elitismo cultural no siempre está correlacionado con opciones estéticas determinadas o con exigencias de calidad; la mayoría de las veces, con discriminaciones en el acceso a los bienes culturales. Por esta razón, toda la oferta cultural de la universidad es gratuita.

Como es sabido, uno de los bienes que exhibe una mayor concentración geográfica en el país es la cultura. En Santiago están las oportunidades y la mayor parte del patrimonio. En el caso de las artes visuales, la capital acapara los centros de formación académica (facultades), la parte más significativa del patrimonio (museos), los circuitos de exhibición y ventas (galerías), los espacios más visibles para la difusión y la reflexión crítica (medios), la parte más significativa del financiamiento (donaciones y presupuesto privado y público ${ }^{4} \mathrm{y}$ la mayor concentración demográfica de artistas en el país. La situación de las artes visuales es perfectamente extrapolable a la música, a la danza, la literatura y otras expresiones del arte.

La universidad ha generado espacio y oportunidades culturales para los habitantes de la Región del Maule, pero también ha creado un ambiente rico en arte y cultura para el desarrollo personal de sus propios estudiantes. Una formación profesional cada día más globa-

4 A modo de ejemplo, mientras el presupuesto del Teatro Municipal de Santiago en el año 2004 fue de $\$ 4.865$ millones, por vía de aporte directo a través del presupuesto nacional, el Área de Cultura de la Región del Maule tuvo una asignación para gastos, en el mismo periodo, de $\$ 4.000 .000$, para atender las actividades de todos los niños de la región. 
lizada exige conocimientos, visiones de mundo y sensibilidades capaces de dar un contenido al fenómeno de la modernidad. La procedencia de una buena parte del estudiantado de ciudades de nivel intermedio o rural, la condición de muchos alumnos de ser primera generación profesional en sus familias y el escaso capital cultural de muchos de ellos, pesan como factores negativos y discriminadores a la hora de su inserción social y laboral.

Gran parte de lo obrado en materia cultural apunta, entonces, a revertir los fenómenos del centralismo cultural -que estructura una desigual y creciente inequidad en el acceso a la cultura-, y al esfuerzo corporativo por compensar el déficit de capital cultural con que ingresa un alto porcentaje de los estudiantes universitarios.

\section{CONTENIDOS DE LA EXTENSIÓN CULTURAL EN LA UNIVERSIDAD DE TALCA}

Las actividades de extensión cultural son desarrolladas a través de la Vicerrectoría de Extensión y Comunicaciones. Forman parte de sus contenidos todas las disciplinas artísticas y culturales, aun cuando se han desarrollado con más profundidad las relacionadas con las artes visuales y la música. Las actividades y los objetivos en estas áreas están orientados básicamente a:

\section{Difusión de las artes}

A través de ciclos anuales de conciertos, exposiciones plásticas, cine, teatro, ferias, entre otras actividades desarrolladas por artistas y elencos tantos externos como de la universidad. Para esto cuenta con los siguientes espacios:

1.1. Centro de Extensión Pedro Olmos, en la Casa Central de la Universidad de Talca. Organizado en tres salas de exposiciones (con un total de $520 \mathrm{~m}^{2}$ ); Sala Pedro Olmos, destinada a exposiciones históricas y antológicas; Sala Giulio di Girólamo, destinada preferentemente a gráfica y fotografía, y Sala de Arte Contemporáneo, para nuevas propuestas estéticas y para artistas de vanguardia, administrada en el contexto de un convenio con el Consejo de la Cultura y las Artes. Además, se cuenta aquí con 
el Salón Abate Molina habilitado para cine (35 mm), teatro y presentaciones de cámara.

1.2. Centro de Extensión de Curicó, con salas de exposiciones (de $200 \mathrm{~m}^{2}$ ) y un teatro con capacidad para 200 personas, habilitado, además, con equipos de proyección para cine $(35 \mathrm{~mm})$.

1.3. Centro de Extensión Profesor Carlos Hojas Alonso, del Campus Lircay (con una superficie de $470 \mathrm{~m}^{2}$ ), especialmente habilitado para las actividades culturales estudiantiles.

1.4. Centro de Extensión Campus Santiago, que cuenta con una sala de exposiciones y una sala de conferencias.

1.5. Arte Comunitario, con un sistema de exposiciones itinerante, pretende hacer llegar a barrios y zonas alejadas distintas colecciones de difusión artística de propiedad de la universidad.

1.6. Feria de Libro del Maule, realizada anualmente desde el año 1995 en convenio con la Cámara Chilena del Libro.

1.7. Radios y televisión: medios de comunicación que permiten difundir la cultura en Talca y la región, a través de la frecuencia de Radio AM (música chilena), Radio FM (música clásica) y canal de televisión por cable-UTT Televisión-, que difunde el quehacer cultural y social.

2. Patrimonio artístico

2.1. Parque de las Esculturas, desarrollado en el Campus Lircay, registra lo más destacado de la escultura chilena en los últimos treinta años. Se han incorporado anualmente obras de los siguientes escultores chilenos: Marta Colvin, Lily Garafulic, Sergio Castillo, Raúl Valdivieso, Federico Assler, Francisco Gazitúa, Aura Castro, Osvaldo Peña, Matías Vial, Samuel Román, José Vicente Gajardo, Mario Irarrázabal, Francisca Cerda, además del artista alemán Waldemar Otto, entre otros.

2.2. Proyecto Pinceladas del Maule: la Universidad de Talca, desde 1995, ha invitado a importantes pintores nacionales a plasmar los paisajes, los personajes y las costumbres de la región. Al término de cada jornada, cada uno deja una de sus obras en esta casa de estudios. Han participado en este proyecto: Ximena Cristi, Sergio Montecino, Augusto Barcia, Hardy Wistuba, Concepción Balmes, Tatiana Alamos, Francisco de la Puente, Alberto Ludwig, 
Flavia Tótoro, Paz Lira, Julien Birke, Francisca Lohmann, Ulrich Wells, Lisy Fox, entre otros.

\subsection{Colecciones}

A través de adquisiciones, donaciones y otras actividades, la universidad ha podido conformar las siguientes colecciones pictóricas:

- Colección Pedro Olmos, integrada por más de 200 dibujos, grabados y pinturas de este artista.

- Colección Giulio di Girólamo, integra unas 300 pinturas, gráfica, diseños gráficos y dibujos.

- Generación de 1940, con más de 50 obras de Fernando Morales Jordán, Carlos Pedraza, Ximena Cristi, Sergio Montecino, Augusto Barcia, entre otros.

- Colección pintura chilena contemporánea, compuesta por unas 100 obras de Francisco de la Puente, Julián Birke, Flavia Tótoro, Tatiana Álamos, José Balmes, Gracia Barrios, Andrés Vió, entre otros pintores.

- Colección Escultura Pequeño Formato, integrada por obras de Aura Castro, Francisca Cerda, José Vicente Gajardo, Matías Vial, Lisie Fox, Juan Díaz Fleming y Sergio Castillo, entre otros artistas.

Actualmente la Universidad de Talca posee un patrimonio artístico de más de 1.000 obras, entre las cuales cabe destacar el mural "Acerca del paisaje interior", del pintor chileno y Premio Nacional de Arte 2001, Rodolfo Opazo, ubicado en el Salón Diego Portales del Campus Lircay.

También se dispone, en comodato, de seis murales de Arturo Gordon y Laureano Guevara, presentados en el stand de Chile en la Exposición Internacional de Sevilla en 1929, los cuales se exhiben en la sala Emma Jauch del Centro de Extensión Pedro Olmos, en la Casa Central.

\section{Editorial}

La universidad creó una editorial, en 1992, como una forma de difundir el trabajo de los académicos, la reflexión y la identidad regional. A la 
fecha ha publicado más de cincuenta libros, además de las revistas de la universidad. Ha definido, además, las colecciones: Ciencia y Tecnología, Identidad Regional del Maule, Estudios Jurídicos y Textos Docentes.

\section{Formación artística}

En 1993, la Universidad de Talca creó una Escuela de Música, como una forma de entregar una formación sistemática a los niños y jóvenes de la región; allí se imparten cursos de teoría musical, violín, guitarra clásica, piano, cello y flauta.

Se han organizado también distintos talleres dirigidos a la comunidad; entre ellos los de Expresión Plástica Infantil, de Dibujo de Figura Humana, de Pintura, de Iniciación a la Escultura y Cerámica, de Literatura, de Poesía, entre otros.

\section{Elencos}

La universidad cuenta con diversos elencos vinculados a la música. Destacan aquí el Coro de Niños, dirigido por la profesora Mirta Bustamante -que ha realizado presentaciones en diversos países de Latinoamérica y ciudades del país-, el Coro Universitario, a cargo del director Guillermo Cárdenas, y las Orquestas Infantil y Juvenil dirigidas por el profesor Américo Giusti.

\section{Premio Iberoamericano de Letras "José Donoso"}

Esta distinción es entregada a un valor destacado de la literatura y letras de Iberoamérica. Un jurado internacional entrega esta distinción, dotada de un premio de US\$20.000. Han recibido este reconocimiento José Emilio Pacheco (México), Beatriz Sarlo (Argentina) e Isabel Allende (Chile).

\section{Financiamiento}

Las actividades de extensión cultural son concebidas en la universidad como bienes públicos; en consecuencia, la institución entrega a estas actividades parte de su infraestructura, aporta el financiamiento de los equipos de gestión y cubre parte de los costos. La Vicerrectoría de 
Extensión y Comunicaciones, a través de sus distintas unidades, gestiona otros recursos por medio de donaciones y fondos concursables.

\section{Convenios}

Para desarrollar su programa de Extensión Cultural, la universidad ha establecido relaciones de trabajo y convenios formales con distintas instituciones y entidades culturales. Dentro de lo primero es posible destacar el trabajo conjunto con el Museo Nacional de Bellas Artes, el Museo de Arte Contemporáneo -dependiente de la Universidad de Chile-, la Pinacoteca de la Universidad de Concepción, la Galería ArteEspacio, entre otros. Se han firmado también convenios con la Fundación Telefónica, el Consejo de las Artes y la Cultura, las municipalidades de Talca y Rancagua, entre otras entidades. 